PROCEEDINGS OF THE

AMERICAN MATHEMATICAL SOCIETY

Volume 125, Number 6, June 1997, Pages 1635-1641

S 0002-9939(97)03938-5

\title{
LOCAL FUNDAMENTAL GROUPS OF ALGEBRAIC VARIETIES
}

\author{
SHREERAM S. ABHYANKAR \\ (Communicated by Ronald M. Solomon) \\ Dedicated to Walter Feit for his Sixty-Fifth Birthday
}

\begin{abstract}
Some progress is made in the calculation of local fundamental
\end{abstract} groups.

\section{INTRODUCTION}

In my 1955-1966 papers [1] to [7], while working on resolution of singularities of algebraic varieties in positive characteristic $p$, I was led to investigate Galois groups of local and global coverings of these varieties. Such Galois groups are the algebraic reflection of local and global (topological) fundamental groups in the complex case. Thus the systems of these Galois groups may be called algebraic fundamental groups. The general surmise which I formed from this investigation was that usually a finite group occurs in a particular characteristic $p$ situation if and only if its maximal prime-to- $p$ quotient occurs in the corresponding complex or characteristic zero situation. In some cases, such as (1) for punctured lines (or more generally, for affine curves), I had stated this surmise as an explicit conjecture. In some other cases such as (2) at normal crossings, and (3) for complements of hyperplanes (or more generally, for complements of hypersurfaces) meeting normally, I had only hinted at it. As we shall see in Section 1, the explicit conjecture has recently been settled by Harbater and Raynaud. This suggests that the hinted conjectures also be made explicit, and I shall do this in Sections 2 and 3 respectively. In Section 4, I shall formulate a local-global conjecture which links the local conjecture of Section 2 with the global conjecture of Section 3. In the various Sections, I shall provide some evidence for the corresponding conjectures.

\section{Punctured Lines}

In 1851, Riemann [18] achieved desingularization of a complex (algebraic) curve $f(X, Y)=0$ by constructing the Riemann Surface of $Y$ as a function of $X$. In this construction, a crucial role is played by the Galois group of $f$ which is the algebraic reflection of the (topological) fundamental group of the multipunctured complex line.

Received by the editors January 5, 1996

1991 Mathematics Subject Classification. Primary 12F10, 14H30, 20D06, 20 E22.

This work was partly supported by NSF grant DMS 91-01424 and NSA grant MDA 904-92$\mathrm{H}-3035$.

(C)1997 American Mathematical Society 
This leads to the definition of the algebraic fundamental group $\pi_{A}\left(L_{k, t}\right)$ of the $t$-punctured affine line $L_{k, t}$ over a field $k$, as the set of all Galois groups of finite Galois extensions of the affine line $L_{k}$ over $k$ which are ramified at most at the $t$ punctures, where $t \geq 0$ is any integer.

For a prime number $p$, let $Q_{t}(p)=$ the set of all quasi- $(p, t)$ groups, i.e., finite groups $G$ such that $G / p(G)$ is generated by $t$ generators, where $p(G)$ is the subgroup of $G$ generated by all of its $p$-Sylow subgroups; members of $Q_{0}(p)$ are called quasi- $p$ groups, and we put $Q(p)=Q_{0}(p)$. We extend this for $p=0$ by agreeing that the identity group 1 is the only 0-Sylow subgroup; note that then $Q_{t}(0)=$ the set of all quasi- $(0, t)$ groups $=$ the set of all finite groups generated by $t$ generators; also note that $0(G)=1$ and $Q_{0}(0)=$ the set of all quasi- $(0,0)$ groups $=$ the set of all quasi- 0 groups $=\{1\}$. Riemann proved that if $k$ is the field of complex numbers then $\pi_{A}\left(L_{k, t}\right)=Q_{t}(0)$. In my 1957 paper [3], as an immediate consequence of the Riemann-Hurwitz genus formula, I proved the following

Result (1.1). If $k$ is an algebraically closed field of char $p \geq 0$ and $0 \leq t \leq 1$ then $\pi_{A}\left(L_{k, t}\right) \subset Q_{t}(p)$.

In my 1957 paper [3], I also made the following conjecture which was settled affirmatively by Harbater and Raynaud in their 1994 papers [13] and [17].

Conjecture (1.2). If $k$ is an algebraically closed field of char $p \geq 0$ and $t \geq 0$ then $\pi_{A}\left(L_{k, t}\right)=Q_{t}(p)$.

Note (1.3). What Harbater proved in [13] was the more general conjecture which I had made in [3] and which says that: if $C_{g, w}=C_{g}$ minus $w+1$ points, where $C_{g}$ is a nonsingular projective curve of genus $g$ over an algebraically closed field $k$ of characteristic $p \geq 0$ and $w$ is a nonnegative integer, then $\pi_{A}\left(C_{g, w}\right)=Q_{2 g+w}(p)$, where $\pi_{A}\left(C_{g, w}\right)$ is again defined to be the set of all Galois groups of unramified finite Galois coverings of $C_{g, w}$. This again concurs with the enough room philosophy according to which a finite group $G$ occurs in a characteristic $p>0$ situation if and only if $G / p(G)$ occurs in the corresponding characteristic zero situation, provided there is "enough room". As an example of "not enough room", for unpunctured $C_{g}$ with $g>0$, in characteristic $p>0$ we do not even get all $G$ which occur in characteristic zero; for a discussion of this see Stevenson [19]. For unpunctured $C_{0}$, regardless of the characterisitc, there are no nontrivial unramified coverings, and so there is "no room".

\section{Normal CROSSINGS}

In 1871, Noether [16] desingularized the curve $f(X, Y)=0$, over a general ground field $k$, by applying a sequence of quadratic transformations to the $(X, Y)$-plane. In 1882, Dedekind [12] obtained desingularization of the curve $f(X, Y)=0$ in one swoop by passing to the integral closure of the residue class ring $k[X, Y] / f(X, Y)$. Out of the three approaches to curve desingularization, only Noether's approach keeps track of the $(X, Y)$-plane. Even then, strictly speaking the $(X, Y)$-plane is changed to a nonsingular surface, but for local considerations it continues to look like a plane.

In 1908, Jung [14] proved local complex surface desingularization by mixing all three ideas. To describe Jung's proof briefly, given a surface $\phi(X, Y, Z)=0$, consider the branch locus $\Delta(X, Y)=0$ as we project it onto the $(X, Y)$-plane, where $\Delta$ is the $Z$-discriminant of $\phi$. Note that if the $Z$-degree of $\phi$ is $n$ then the 
projection is $n$ to 1 except at the points of $\Delta=0$ above which there are less than $n$ points of the surface. The branch locus actually consists of certain irreducible components of $\Delta=0$. At any rate, in view of Noether's curve desingularization, after applying a finite number of quadratic transformations to the $(X, Y)$-plane, we may assume that $\Delta=0$ has only normal crossings. If a point of the surface projects outside $\Delta=0$ then it is automatically simple, whereas if it projects into a normal crossing of $\Delta=0$ then, as observed by Jung, it is easy to resolve because of the topological fact that the local fundamental group at a normal crossing is abelian.

In the failure part of my Ph.D. Thesis [1] published in 1955, I algebracized the above topological fact by considering the local algebraic fundamental group $\pi_{A}^{L}\left(N_{k, t}^{d}\right)$ at a $t$-fold normal crossing.

To define this let $P \in W \subset V$ where $P$ is a simple point of an irreducible algebraic variety $V$ of dimension $d \geq 2$ over the ground field $k$, and $W$ is a subvariety of $V$ having a $t$-fold normal crossing at $P$, i.e., in the local $\operatorname{ring} R$ of $P$ on $V$, the ideal of $W$ is of the form $x_{1} \ldots x_{t} R$ where $x_{1}, \ldots, x_{d}$ is a basis of the maximal ideal $M(R)$ of $R$. Let $K=$ the function field $k(V)$, i.e., $K=$ the quotient field of $R$. Let $\Omega^{*}$ be the set of all finite Galois extensions $K^{*}$ of $K$, in a fixed algebraic closure of $K$, such that $x_{1} R, \ldots, x_{t} R$ are the only height one prime ideals of $R$ which are possibly ramified in $K^{*}$. Let $\widehat{K}$ be the quotient field of the completion of $R$. For every $K^{*} \in \Omega^{*}$ let $\widehat{K}^{*}$ be the quotient field of the completion of a localization of the integral closure of $R$ in $K^{*}$ at a maximal ideal in the said integral closure. Then $\pi_{A}^{L}(V, W, P)$, or briefly $\pi_{A}^{L}\left(N_{k, t}^{d}\right)$ with $N_{k, t}^{d}=(V, W, P)$, is defined to be the set of all Galois groups $\operatorname{Gal}\left(\widehat{K}^{*}, \widehat{K}\right)$ as $K^{*}$ varies in $\Omega^{*}$. In other words, $\pi_{A}^{L}(V, W, P)$ is the set of all local Galois groups of finite Galois coverings of $V$ at $P$ whose branch locus at $P$ is contained in the subvariety $W$ having a $t$-fold normal crossing.

For $p=$ a prime number or 0 , let $P_{t}(p)=$ the set of all $(p, t)$-groups, i.e., the set of all finite groups $G$ such that $G / p(G)$ is an abelian group generated by $t$ generators. Note that then $P_{t}(p)=Q_{t}(p)$ for $0 \leq t \leq 1$, but $P_{t}(p)$ is much smaller than $Q_{t}(p)$ for $t>1$. In [1] I explicitly proved the following

Result (2.1). If $k$ is an algebraically closed field of char $p \geq 0$ and $t>0$ then $\pi_{A}^{L}\left(N_{k, t}^{d}\right) \subset P_{t}(p)$.

Moreover, in [1] I implicitly made the following

Conjecture (2.2). If $k$ is an algebraically closed field of char $p \geq 0$ and $t>0$ then $\pi_{A}^{L}\left(N_{k, t}^{d}\right)=P_{t}(p)$.

Remark (2.3). The proof of Result (2.1) uses Zariski's [20] Purity of Branch Locus. Since Nagata [15] has proved Purity for any regular local ring, Result (2.1) remains true for $\pi_{A}^{L}(R, I, M(R))$ where $R$ is any regular local ring of dimension $d \geq 2$, $k$ is the residue field $R / M(R)$, and $I=x_{1} \ldots x_{t} R$ for some basis $x_{1}, \ldots, x_{d}$ of the maximal ideal $M(R)$ of $R$. The definition of $\pi_{A}^{L}(R, I, M(R))$ is exactly as above, i.e., it is the set of all Galois groups $\operatorname{Gal}\left(\widehat{K}^{*}, \widehat{K}\right)$ as $K^{*}$ varies in $\Omega^{*}$. Note that now char $k$ is allowed to be different from char $R$. It is rather likely that Conjecture (2.2) remains valid in this general case. In the equicharacteristic case, i.e, when char $k=$ char $R$, we may still write $\pi_{A}^{L}\left(N_{k, t}^{d}\right)=\pi_{A}^{L}(R, I, M(R))$ with $N_{k, t}^{d}=(R, I, M(R))$. In the unequicharacteristic case, i.e, when char $k \neq$ char $R$, we may write $\pi_{A}^{L}\left(U_{k, t}^{d}\right)=\pi_{A}^{L}(R, I, M(R))$ with $U_{k, t}^{d}=(R, I, M(R))$. 
Remark (2.4). If we extend the meaning of $\pi_{A}^{L}\left(N_{k, t}^{d}\right)$ to $t=0$ in an obvious manner, then by Purity, regardless of the characteristic, we would have $\pi_{A}^{L}\left(N_{k, t}^{d}\right)=\{1\}$. However, for $p>0$, the family $P_{0}(p)$ is quite large as it contains all finite simple groups whose orders are divisible by $p$. This is why in (2.1) and (2.2) we have stressed the condition that $t>0$.

Remark (2.5). In [1], in support of Conjecture (2.2) I wrote down an example of a surface with branch locus having a simple point but with unsolvable local Galois group. A slightly simplified avatar of the said example is given by the surface $F=0$ over an algebraically closed ground field $k$ of characteristic $p>0$ where $F=Z^{n}+Y Z+X \quad$ with $0<p<n \equiv 1 \bmod p$. Note that $\operatorname{Disc}_{Z}(F)=X^{n-1}$ where $\operatorname{Disc}_{Z}(F)$ is the $Z$-discriminant of $F$, and hence we have a case of $N_{k, t}^{d}$ with $d=2$ and $t=1$. In [1], I showed that for $n=p+1$ and $p=5$ the Galois group $\operatorname{Gal}(F, k((X, Y)))$ is unsolvable, and, for $n=p+1$ and any $p>0$, its order is divisible by $p(p+1)$ and hence it is quite complicated. Thus I concluded that Jung's surface desingularization method could not be used for nonzero characteristic. This is why I have called [1] the failure part of my Thesis. In the success part [2] published in 1956, I proved surface desingularization in nonzero characteristic by other means. Later on, in 1965 [6] I extended it to arithmetic surfaces, and in 1966 [7] to three-dimensional varieties over ground fields of nonzero characteristic. Now, having learnt some more group theory, I can show that, for $n=1+q+q^{2}+\cdots+$ $q^{m-1}$ where $q>1$ is any power of $p$ and $m>1$ is any integer, we actually have $\operatorname{Gal}(F, k((X, Y)))=\operatorname{PGL}(m, q)$. Clearly this follows by taking $R=k[[X, Y]]$ in the following slightly more general result whose proof, given in (5.3) of [11], occurred to me while listening to a masterful lecture of Walter Feit in the May 1995 Ohio-State Conference in honor of his Sixty-Fifth Birthday.

Result (2.6). Let $n=1+q+q^{2}+\cdots+q^{m-1}$ where $q>1$ is any power of a prime number $p$ and $m>1$ is any integer. Let $K$ be the quotient field of a regular local domain $R$ of dimension $d \geq 2$ with $M(R)=\left(X, Y, Y_{3}, \ldots, Y_{d}\right) R$ such that $G F(q)$ is a subfield of $R$. Let $F(Z)=Z^{n}+Y Z+X$ and $\Phi(Z)=Z^{q^{m}-1}+Y Z^{q-1}+X$. Then $\operatorname{Gal}(F, K)=P G L(m, q)$ and $\operatorname{Gal}(\Phi, K)=G L(m, q)$ in a natural manner in such a way that $\operatorname{Gal}(F, K)$ is the image of $G a l(\Phi, K)$ under the canonical map of $G L(m, q)$ onto PGL $(m, q)$. [Note that $\operatorname{Disc}_{Z}(F)=X^{n-1}$ and $\operatorname{Disc}_{Z}(\Phi)=-(-X)^{q^{m}-2}$.]

Remark (2.7). Let $k$ be an algebraically closed field of characteristic $p>0$, let $q>1$ be any power of $p$ and let $m>1$ be any integer. In support of Conjecture (2.2), the above Result (2.6) shows that the groups $\operatorname{PGL}(m, q)$ and $\operatorname{GL}(m, q)$ belong to $\pi_{A}^{L}\left(N_{k, t}^{d}\right)$ for $t=1$ and hence for all $t>0$ with $t \leq d$. In my 1958 paper [4], I had also shown that, in case $p=2$, the symmetric group $S_{m}$ belongs to $\pi_{A}^{L}\left(N_{k, t}^{d}\right)$ for $t=1$ and hence for all $t>0$ with $t \leq d$.

Note (2.8). Clearly Conjecture (2.2) concurs with the "enough room philosophy" propounded in Note (1.3). An example of "not enough room" is provided by Remark (2.4). Yet another example of "not enough room" is obtained by extending the meaning of $\pi_{A}^{L}\left(N_{k, t}^{d}\right)$ to $d=1$ in an obvious manner; now, for $p=0$, by Newton's Theorem, $\pi_{A}^{L}\left(N_{k, 1}^{1}\right)$ consists of all finite cyclic groups; however, for $p>0$, by Generalized Newton's Theorem, every member of $\pi_{A}^{L}\left(N_{k, 1}^{1}\right)$ is solvable and hence $\pi_{A}^{L}\left(N_{k, 1}^{1}\right)$ is much smaller than $P_{1}(p)$. Reverting to $d \geq 2$, we note that the evidence for Conjecture (2.2) provided in Remark (2.7) is really for $d=2$ with $d-2$ dummy 
variables. In case of characteristic zero, it is easy to provide evidence which is genuine for any $d$. Indeed, in case of characteristic zero, by adjoining roots of the variables (i.e., of a minimal set generators of the maximal ideal of the regular local ring), we easily see that $\pi_{A}^{L}\left(N_{k, t}^{d}\right)=P_{t}(0)=$ the set of all finite abelian groups generated by $t$ generators.

\section{Complements of hyperplanes}

Let $L_{k}^{d}$ be the $d$-dimensional affine space over a field $k$ with $d \geq 2$, and let $L_{k, t}^{d}=L_{k}^{d}-H$ where $H$ is a union of $t$ (distinct) hyperplanes in $L_{k}^{d}$ such that $H \cup H_{\infty}$ (where $H_{\infty}$ is the hyperplane at infinity) has only normal crossings, i.e., $H \cup H_{\infty}$ has a $t(P)$-fold normal crossing at every point $P$ of $H \cup H_{\infty}$ for some positive integer $t(P) \leq d$ depending on $P$. Let $\Omega^{\prime}$ be the set of all finite Galois extensions $K^{\prime}$ of $k\left(L_{k}^{d}\right)$, in a fixed algebraic closure of $k\left(L_{k}^{d}\right)$, such that the prime ideals of the various irreducible components of $H$ are the only height one prime ideals in the affine coordinate ring $k\left[L_{k}^{d}\right]$ which are possibly ramified in $K^{\prime}$. Then the algebraic fundamental group $\pi_{A}\left(L_{k, t}^{d}\right)$ is defined to be the set of all Galois groups $\operatorname{Gal}\left(K^{\prime}, k\left(L_{k}^{d}\right)\right)$ as $K^{\prime}$ varies in $\Omega^{\prime}$. In my 1959-1960 paper [5] I explicitly proved the following

Result (3.1). If $k$ is an algebraically closed field of char $p \geq 0$ and $t \geq 0$ then $\pi_{A}\left(L_{k, t}^{d}\right) \subset P_{t}(p)$.

Moreover, in [5] I implicitly made the following

Conjecture (3.2). If $k$ is an algebraically closed field of char $p \geq 0$ and $t \geq 0$ then $\pi_{A}\left(L_{k, t}^{d}\right)=P_{t}(p)$.

Remark (3.3). A Bertini argument (see [5]) shows that, in case of $0 \leq t \leq 1$, Conjectures (1.2) and (3.2) are equivalent to each other.

Note (3.4). To state a stronger version of (3.1) proved in [5], let $V$ be the $d$ dimensional projective space over an algebraically closed field $k$ of characteristic $p \geq 0$ with $d \geq 2$, let $t \geq 0$ be an integer, and let $W$ be a hypersurface in $V$ having $t+1$ irreducible components $W_{1}, W_{2}, \ldots, W_{t+1}$. Assume that at every point $P$ of it, $W$ has an $s$-fold normal crossing for some positive integer $s$ which may depend on $P$; note that then each $W_{i}$ is nonsingular. Let $P_{t}^{*}(p)$ be the set of all finite groups $G$ such that $G / p(G)$ is an abelian group generated by $t+1$ generators $a_{1}, \ldots, a_{t+1}$ satisfying the relation $\prod_{1<i<t+1} a_{i}^{e(i)}=1$ where $e(i)$ is the degree of $W_{i}$. Then the said stronger version of $(3 . \overline{1})$ proved in [5] says that $\pi_{A}(V-W) \subset P_{t}^{*}(p)$ where $\pi_{A}(V-W)$ is again defined to be the set of all Galois groups of unramified finite Galois coverings of $V-W$. In [5] it is also shown that for $p=0$ we have the equality $\pi_{A}(V-W)=P_{t}^{*}(p)$. In concurrence with the "enough room philosophy" propounded in Note (1.3), the corresponding version of (3.2) would conjecture that also for $p>0$ we have the equality $\pi_{A}(V-W)=P_{t}^{*}(p)$.

\section{Plane sections}

As said in Remark (2.5), in my 1955 paper [1] I found unsolvable surface coverings above a simple point of the branch locus. By taking plane sections of these surface coverings, in my 1957 paper [3] I was led to write down explicit equations of unramified coverings of $L_{k}$ and $L_{k, 1}$ over a field $k$ of characteristic $p>0$, and this is 
what motivated Conjecture (1.2). In the 1992-1995 papers [8] to [10], I computed the Galois groups of some of these equations. For instance, in (3.2) of [9] I proved the following

Result (4.1). Let $n=1+q+q^{2}+\cdots+q^{m-1}$ where $q>1$ is any power of a prime number $p$ and $m>1$ is any integer. Let $k$ be an overfield of $G F(q)$. Let $\widetilde{F}(Z)=Z^{n}+X Z+X$ and $\widetilde{\Phi}(Z)=Z^{q^{m}-1}+X Z^{q-1}+X$. Then $\operatorname{Gal}(\widetilde{F}, k(X))=$ $P G L(m, q)$ and $\operatorname{Gal}(\widetilde{\Phi}, k(X))=G L(m, q)$ in a natural manner in such a way that $\operatorname{Gal}(\widetilde{F}, k(X))$ is the image of $\operatorname{Gal}(\widetilde{\Phi}, k(X))$ under the canonical map of $G L(m, q)$ onto $P G L(m, q)$. [Note that $\operatorname{Disc}_{Z}(\widetilde{F})=X^{n-1}$ and $\operatorname{Disc}_{Z}(\widetilde{\Phi})=-(-X)^{q^{m}-2}$.]

Again let $L_{k}^{d}$ be the $d$-dimensional affine space over a field $k$ with $d \geq 2$, and let us consider $L_{k, 1}^{d}=L_{k}^{d}-L_{k}^{d-1}$ where $L_{k}^{d-1}$ is a hyperplane in $L_{k}^{d}$. Let $\Omega^{\prime}$ be the set of all finite Galois extensions $K^{\prime}$ of $k\left(L_{k}^{d}\right)$, in a fixed algebraic closure of $k\left(L_{k}^{d}\right)$, such that the prime ideal of $L_{k}^{d-1}$ is the only height one prime ideal in $k\left[L_{k}^{d}\right]$ which is possibly ramified in $K^{\prime}$. Let $\Omega^{\prime *}$ be the set of all $K^{\prime *} \in \Omega^{\prime}$ for which there exists an affine line $L_{k}$ in $L_{k}^{d}$ which meets $L_{k}^{d-1}$ in a point $P=L_{k}^{d-1} \cap L_{k}$ such that the integral closures in $K^{\prime *}$ of the local rings of $P$ and $L_{k}$ on $L_{k}^{d}$ are local. Let us define local-global algebraic fundamental group $\pi_{A}^{L G}\left(L_{k, 1}^{d}\right)$ to be the set of all Galois groups $\operatorname{Gal}\left(K^{\prime *}, k\left(L_{k}^{d}\right)\right)$ as $K^{\prime *}$ varies in $\Omega^{\prime *}$, and about it let us offer the following

Conjecture (4.2). If $k$ is an algebraically closed field of char $p \geq 0$ then $\pi_{A}^{L G}\left(L_{k, 1}^{d}\right)$ $=P_{1}(p)$.

Remark (4.3). Conjecture (4.2) is obviously stronger than the $t=1$ case of each of the three Conjectures (1.2), (2.2) and (3.2). All these are trivially true for $p=0$. In the nontrivial case of an algebraically closed field $k$ of characteristic $p>0$, equationally speaking, given any $G \in Q_{1}(p)$, Conjecture (4.2) asks us to find a monic polynomial $\Psi(Z)$ in $Z$ with coefficients in $A=k\left[X, Y, Y_{3}, \ldots, Y_{d}\right]$ such that $X A$ is the only height one prime ideal on $A$ which is possibly ramified in the splitting field $K^{\prime *}$ of $\Psi(Z)$ over $K=k\left(X, Y, Y_{3}, \ldots, Y_{d}\right)$ and upon letting $\widetilde{\Psi}(Z)$ be obtained by putting $Y=Y_{3}=\cdots=Y_{d}=0$ in $\Psi(Z)$ we have $\operatorname{Gal}(\Psi, K)=$ $\operatorname{Gal}(\Psi, \widehat{K})=\operatorname{Gal}(\widetilde{\Psi}, \widetilde{K})=G$ where $\widehat{K}=k\left(\left(X, Y, Y_{3}, \ldots, Y_{d}\right)\right)$ and $\widetilde{K}=k(X)$. If in doing this we can also arrange that $\operatorname{Disc}_{Z}(\Psi)=c X^{e}$ with $0 \neq c \in k$ and integer $e \geq 0$ then it would be like sugar in the milk or icing on the cake. Given any $q>1$ which is a power of $p$ and any integer $m>1$, in view of Results (2.6) and (4.1), for $G=\operatorname{PGL}(m, q)$ we can achieve this by taking $\Psi(Z)=Z^{n}+(Y+X) Z+X$ with $n=1+q+q^{2}+\cdots+q^{m-1}$, and for $G=\mathrm{GL}(m, q)$ we can achieve it by taking $\Psi(Z)=Z^{q^{m}-1}+(Y+X) Z^{q-1}+X$.

Note (4.4). Again we note that the evidence for Conjecture (4.2) provided in Remark (4.3) is really for $d=2$ with $d-2$ dummy variables. This is not surprising because Conjecture (4.2) is essentially 2-dimensional in nature. A truly higher dimensional version of Conjecture (4.2) remains to be found.

\section{REFERENCES}

[1] S. S. Abhyankar, On the ramification of algebraic functions, American Journal of Mathematics 77 (1955), 572-592. MR 17:193c

[2] S. S. Abhyankar, Local uniformization on algebraic surfaces over ground fields of characteristic $p \neq 0$, Annals of Mathematics 63 (1956), 491-526. MR 17:1134d 
[3] S. S. Abhyankar, Coverings of algebraic curves, American Journal of Mathematics 79 (1957), 825-856. MR 20:872

[4] S. S. Abhyankar, On the ramification of algebraic functions Part II, Transactions of the American Mathematical Society 89 (1958), 310-324. MR 20:5204

[5] S. S. Abhyankar, Tame coverings and fundamental groups of algebraic varieties Parts I to VI, American Journal of Mathematics 81-82 (1959-1960), 46-94 of vol 81 and 120-190 + 341-388 of vol 82. MR 22:1578; MR 21:3428

[6] S. S. Abhyankar, Resolution of singularities of arithmetical surfaces, Arithmetical Algebraic Geometry (1965), 111-152. MR 34:171

[7] S. S. Abhyankar, Resolution of Singularities of Embedded Algebraic Surfaces, Academic Press, 1966. MR 36:164

[8] S. S. Abhyankar, Galois theory on the line in nonzero characteristic, Bulletin of the American Mathematical Society 27 (1992), 68-133. MR 94a:12004

[9] S. S. Abhyankar, Nice equations for nice groups, Israel Journal of Mathematics 88 (1994), 1-24. MR 96f: 12003

[10] S. S. Abhyankar, Mathieu group coverings and linear group coverings, Contemporary Mathematics 186 (1995), 293-319. CMP 96:01

[11] S. S. Abhyankar, Projective polynomials (To Appear).

[12] R. Dedekind and H. Weber, Theorie der alg. Funktionen einer veränderlichen, Crelle Journal 92 (1882), 181-290.

[13] D. Harbater, Abhyankar's conjecture on Galois groups over curves, Inventiones Mathematicae 117 (1994), 1-25. MR 95i:14029

[14] H. W. E. Jung, Darstellung der Funktionen eines algebraischen Körpers zweier Veränderlichen $x, y$ in der Umgebung einer Stelle $x=a, y=b$, Crelle Journal 133 (1908), 289-314.

[15] M. Nagata, On the purity of branch loci in regular local rings, Illinois Journal of Mathematics 3 (1959), 328-333. MR 21:5660

[16] M. Noether, Über die alg. Functionen einer und zweier Variabeln, Göttinger Nachrichten (1871).

[17] M. Raynaud, Revêtment de la droit affine en charactéristic $p>0$ et conjecture d'Abhyankar, Inventiones Mathematicae 116 (1994), 425-462. MR 94m:14034

[18] B. Riemann, Grundlagen für eine allgemeine Theorie der Functionen einer veränderlichen complexen Grösse, Inauguraldissertation, Göttingen (1851), 1-48.

[19] K. F. Stevenson, Galois groups of unramified covers of projective curves in characteristic $p$, Journal of Algebra 44, (To Appear).

[20] O. Zariski, On the purity of the branch locus of algebraic functions, Proc. Nat. Acad. Sci. U.S.A. 44 (1958), 791-796. MR 20:2344

Department of Mathematics, Purdue University, West Lafayette, Indiana 47907

E-mail address: ram@cs.purdue.edu 